\title{
Optimal Motion of an Articulated Body in a Perfect Fluid
}

\author{
Eva Kanso and Jerrold E. Marsden
}

\begin{abstract}
An articulated body can propel and steer itself in a perfect fluid by changing its shape only. Our strategy for motion planning for the submerged body is based on finding the optimal shape changes that produce a desired net locomotion; that is, motion planning is formulated as a nonlinear optimization problem.
\end{abstract}

\section{INTRODUCTION}

We study the locomotion of an articulated body that can undergo shape changes and is immersed in a perfect fluid, as shown in Figure 1. Starting from rest, the articulated body can propel and steer itself in an irrotational fluid by changing its shape. The goal of this work is to investigate the optimal shape changes that produce a desired net locomotion.

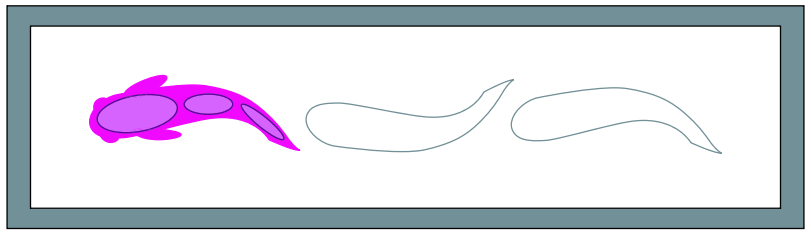

Fig. 1. Fish swimming in an infinite potential flow: the body of the fish is modeled as a system of articulated solid bodies.

Early efforts in developing mathematically-sound models of swimming can be attributed to the work of Gray, Childress, Lighthill, Taylor and Wu; see [3], [10], [21] and [23]. Interest re-emerged over the past few years to understand the mechanics of fish swimming and thereby enable novel engineering applications such as the design of biologicallyinspired vehicles that move and steer by changes of shape rather than by direct propulsion. For recent experimental studies of the shape kinematics of biological fish and its interation with the surrounding fluid, see, for example, [9], [14], and [22]. See also [7] and [18] for their fundamental work on the mathematical formulation of aquatic locomotion using tools from geometric mechanics.

a) Swimming in Potential Flow: In [6], we modeled the fish as an articulated body made of three rigid links, and formulated the equations governing its motion in potential flow using tools from geometric mechanics; namely, we established the trajectories of the net rigid motion $g(t)$ as geometric phases, or holonomy, over closed curves in the shape space (the space of allowable relative rotations $\theta_{1}(t)$ and $\theta_{2}(t)$ between the links). We showed under these idealized conditions, i.e., in the absence of a vortex shedding mechanism, that the fish can propel and steer itself by changing its shape only. This result is important because,

Control \& Dynamical Systems, California Institute of Technology, Pasadena, CA 91125, ekanso@cds. caltech.edu contrary to some common beliefs, it demonstrates that the forces and moments applied on the fish body by shed vortices are not solely responsible for the net locomotion. The net locomotion in potential flow occurs due to the transfer of momentum between the solid and the fluid: starting from rest, the articulated body changes its shape by applying internal torques at its joints. This shape actuation sets the surrounding fluid into motion, and the coupling between the shape dynamics and the surrounding fluid causes a net locomotion of the solid.

b) Motion Planning: There is a need to establish a rigorous foundation for the selection of patterns of shape changes that produce a desired net locomotion. Motion planning and control of self-propelled underwater robotic vehicles have been the subject of several recent studies, as in [17], [2], [13] and [16]; see also references therein. These studies however address local motion planning and make the restrictive and unrealistic assumption of small shape changes, hence the need for alternative global methods. In the present work, the assumption of small shape changes is not required, and the problem of motion planning is formulated as an optimization problem that maximizes/minimizes certain cost functions. That is, we ask the question: "what are the optimal shape changes that achieve a desired net locomotion?" (see also [12] for analogous ideas for the falling cat problem). This approach is capable of capturing the complex behavior of biological fish. Indeed, it is well known that biological fish change their behavior depending on the conditions in which they swim. When swimming peacefully, their concern is to minimize their energy cost but, if attacked by a predator, their energy concerns become secondary as they speed up to escape.

c) Discrete Optimal Control: We use a new approach proposed by [5] for the optimal control of mechanical systems. The main idea is to discretize the Lagranged'Alembert principle directly instead of the associated forced Euler-Lagrange equations. The resulting discrete equations then serve as constraints for the optimization of the given cost functional. This approach respects, by construction, the conserved quantities in the mechanical system, and is particularly useful for the fish problem because the total momentum is conserved. The setting of the discrete optimal control method within the general optimization framework is discussed in [5]. For a different approach to motion planning of the three-link fish, see [11] .

d) Organization of the Paper: First, §II describes the general setting of the problem. We write the kinetic energy of the solid-fluid system in $\S$ III, and we formulate the dynamics using the Lagrange-D'Alembert variational principle in $\S \mathrm{IV}$. 
Motion planning is established as a problem in discrete optimal control in $\S \mathrm{V}$. The implementation and numerical results are presented in $\S \mathrm{VI}$.

\section{PROBLEM Description}

e) Setting: Consider an articulated body formed of rigid links and immersed in an infinitely large volume of an incompressible fluid which is at rest at infinity, see Figure 1. Assume that the fluid particles may slip along the boundaries of the solid but do not allow cavities to form in the fluid nor at the interface. It is well-known in fluid mechanics that, under these conditions, the equations governing the motion of the solid in an irrotational fluid can be written without explicitly incorporating the ambient fluid (see, for example, [6] for details). That is, the configuration space of the solid-fluid system can be identified with that of the submerged solid only.

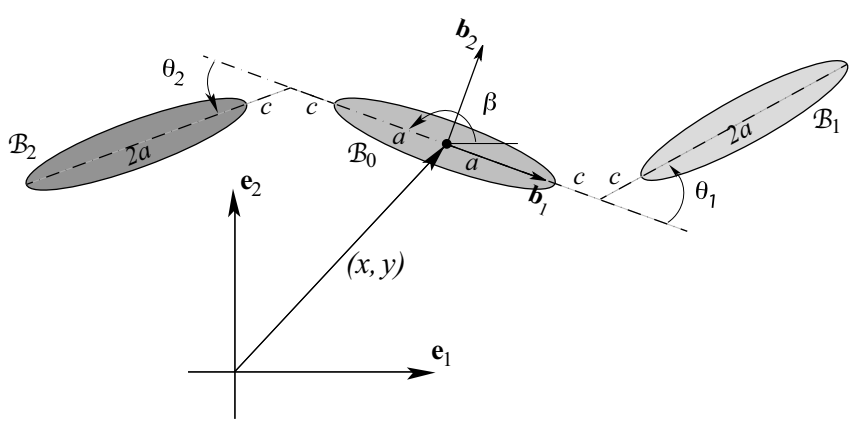

Fig. 2. A three-link mechanism submerged in a perfect fluid.

f) The Articulated Body: For concreteness, let the articulated body and fluid occupy the two-dimensional plane $M \equiv \mathbb{R}^{2}$ and let $\mathcal{F}$ be the region (or subset) of $M$ occupied by the fluid. We consider the articulated body to be formed of 3 neutrally-buoyant, rigid ellipses $\mathcal{B}_{i}, i=0,1,2$, connected together via hinge joints. More specifically, we assume that the ellipses are made of a homogeneous material with density $\rho_{s}$ equal to that of the fluid $\rho_{f}$, and $\mathcal{B}_{i}$ has dimension $a_{i}$ along its major axes and $b_{i}$ along its minor axes. The hinge joints allow the linked ellipses to rotate relative to each other but constrain their translational motion. We further assume that the joints are equipped with motors that generate torques $\tau_{1}$ and $\tau_{2}$ to set $\mathcal{B}_{1}$ and $\mathcal{B}_{2}$ in motion relative to $\mathcal{B}_{0}$. To study the motion of the 3-link body, it is convenient to introduce an orthonormal inertial frame $\left\{\mathbf{e}_{k}\right\}, k=1,2,3$, where $\left\{\mathbf{e}_{1}, \mathbf{e}_{2}\right\}$ span the plane $M$ and $\mathbf{e}_{3}$ is the unit normal to $M$. Likewise, a body-fixed frame $\left\{\mathbf{b}_{k}\right\}$ is attached at the center of mass of each of the three solid links.

g) The Shape Space: Let $\theta_{1}$ and $\theta_{2}$ denote the rigid rotations of $\mathcal{B}_{1}$ and $\mathcal{B}_{2}$ relative to $\mathcal{B}_{0}$ as shown in Figure 2. We refer to the space of admissible relative rotations $\left(\theta_{1}, \theta_{2}\right)$ as the shape space $\mathfrak{X}$. Clearly, $\left(\theta_{1}, \theta_{2}\right)$ completely determine the shape of the three-link body but not its position and orientation relative to the inertial frame $\left\{\mathbf{e}_{k}\right\}$. The latter information is given by $(\beta, x, y)$, where $\beta$ denotes the orientation of $\mathcal{B}_{0}$ and $(x, y)$ the position of its mass center, see Figure 2. Hence, the configuration of the articulated body can be fully described using $\left(\theta_{1}, \theta_{2}, \beta, x, y\right)$.

h) The Bundle Structure: Let $\mathcal{R}$ denote the space of all admissible configurations. It can be shown that $\mathcal{R}$ forms a principal bundle over the shape space $\mathfrak{X}$, see [6]. That is, roughly speaking, at each point $\left(\theta_{1}, \theta_{2}\right)$ of the shape space, one has a copy of the group of rigid motions $\operatorname{SE}(2)$ parameterized by $(\beta, x, y)$, see Figure 3 .

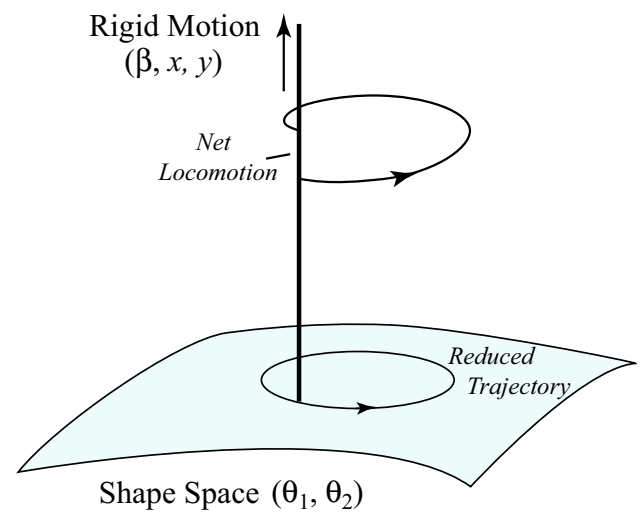

Fig. 3. The configuration space $\mathcal{R}$ has the structure of a principal bundle over the shape space $\mathfrak{X}$, which allows the net locomotion $g$ to be cast as a geometric phase, or holonomy, over closed trajectories traced by the shape variables $\left(\theta_{1}, \theta_{2}\right)$.

This geometric picture is very convenient to address the locomotion problem; namely, the net rigid motion $(\beta, x, y)$ achieved as a result of $\left(\theta_{1}, \theta_{2}\right)$ tracing a closed trajectory in the shape space. In this work, we investigate the problem of motion planning or finding the most efficient trajectories in the shape space that produce a desired net locomotion.

i) Velocities of the Solid Links: Let $\Omega_{i}$ and $v_{i}$ be, respectively, the angular and translational velocities of $\mathcal{B}_{i}$ expressed relative to $\mathcal{B}_{i}$-fixed frame. For conciseness, we introduce $\xi_{i}$ such that $\xi_{i}^{T}=\left(\Omega_{i}, v_{i}\right)^{T}$. For example, one has $\xi_{0}^{T}=\left(\Omega_{0}, v_{0}\right)^{T}$ where

$$
\Omega_{0}=\dot{\beta}, \quad v_{0}=\left(\begin{array}{c}
\dot{x} \cos \beta+\dot{y} \sin \beta \\
-\dot{x} \sin \beta+\dot{y} \cos \beta
\end{array}\right) .
$$

This notation is consistent with the group theoretic notation. For a brief review of the planar rigid motion group $\mathrm{SE}(2)$ and its Lie algebra $\mathfrak{s e}(2)$, see, e.g., [7, Chapter 2].

j) The Dynamics: The equations governing the dynamics of the submerged three-link fish (summarized in (10) and (11)) are derived in [6] and recalled in $\S$ III and $\S I V$. Equations (10) and (11) can be written explicitly in terms of the configuration variables $\left(\theta_{1}, \theta_{2}, \beta, x, y\right)$ and the forcing torques (also called actuators) $\tau_{1}$ and $\tau_{2}$ to give a system of five second-order differential equations. The resulting second-order system can be transformed into a system of ten first-order equations of the form $\dot{z}=f(z, w)$ in terms of the state variables $z=\left(\theta_{1}, \theta_{2}, \beta, x, y, \dot{\theta}_{1}, \dot{\theta}_{2}, \dot{\beta}, \dot{x}, \dot{y}\right)$ and the control variables $w=\left(0,0,0,0,0, \tau_{1}, \tau_{2}, 0,0,0\right)$. That is, Equations (10) and (11) could be written in the well-known state-space representation $\dot{z}=f(z, w)$ and $g=(\beta, x, y)$ (the net locomotion $g$ is the output). However, the discrete 
optimal control approach does not require such rewriting of the equations.

\section{KINETIC ENERGY}

The kinetic energy $T$ of the solid-fluid system can be written as the sum of the energies of the solid links $T_{\mathcal{B}_{i}}$ and the energy of the fluid $T_{\mathcal{F}}$; namely,

$$
T=\sum_{i=0}^{2} T_{\mathcal{B}_{i}}+T_{\mathcal{F}} .
$$

k) Kinetic Energy of the Solid Ellipses: The kinetic energy $T_{\mathcal{B}_{i}}$ can be written in the form

$$
T_{\mathcal{B}_{i}}=\frac{1}{2} \xi_{i}^{T} \mathbb{I}_{i}^{s} \xi_{i}, \quad i=0,1,2 .
$$

Here, $\mathbb{I}_{i}^{s}$ is a $3 \times 3$ diagonal matrix with diagonal entries $\left(I_{i}, m_{i}, m_{i}\right)$ where $I_{i}=m_{i}\left(a_{i}^{2}+b_{i}^{2}\right) / 4$ is the moment of inertia of $\mathcal{B}_{i}$ and $m_{i}=\rho_{s} \pi a_{i} b_{i}$ is its mass. It is important to recall that the links are neutrally buoyant, that is, $\rho_{s}=\rho_{f}$ and that the body-fixed frames are placed at the respective mass centers.

l) Kinetic Energy of the Fluid: The kinetic energy of the fluid $T_{\mathcal{F}}$ is given in spatial representation by

$$
T_{\mathcal{F}}=\frac{1}{2} \int_{\mathcal{F}} \rho_{f}|u|^{2} \mathrm{~d} a,
$$

where $u$ is the spatial velocity field of the fluid and $\mathrm{d} a$ is the standard area element on $\mathbb{R}^{2}$. For potential flow, the fluid velocity can be written as the gradient of a potential function $u=\nabla \phi$, where the potential $\phi$ is the solution to Laplace's equation $\Delta \phi=0$ subject to the boundary conditions

$$
\left\{\begin{array}{ll}
\nabla \phi \cdot n_{i}=\left(v_{i}+\Omega_{i} \times X_{i}\right) \cdot n_{i} & \text { on } \partial \mathcal{B}_{i} \\
\nabla \phi=0 & \text { at } \infty
\end{array} .\right.
$$

Here, $n_{i}$ is a unit normal to $\partial \mathcal{B}_{i}$ and $X_{i}$ is the position vector of a point on $\partial \mathcal{B}_{i}$ relative to the respective mass center. Under these conditions, one can show following a standard procedure (see, for example, [6] and references therein) that $T_{\mathcal{F}}$ of (4) can be rewritten as

$$
T_{\mathcal{F}}=\frac{1}{2} \xi_{i}^{T} \mathbb{I}_{i j}^{f} \xi_{j}, \quad i, j=0,1,2
$$

The $3 \times 3$ added inertia matrices $\mathbb{I}_{i j}^{f}$ depend on the geometry and relative configurations $\left(\theta_{1}, \theta_{2}\right)$ of the submerged ellipses and are of the form

$$
\mathbb{I}_{i j}^{f}=\left(\begin{array}{l|l}
J_{i j} & d_{i j}^{T} \\
\hline d_{i j} & M_{i j}
\end{array}\right),
$$

where $J_{i j}$ are scalars that represent added moments of inertia due to the presence of the fluid, $M_{i j}$ are $2 \times 2$ symmetric matrices that represent added masses, and $d_{i j}$ are $2 \times 1$ arrays that reflect a coupling between the angular and translational motions due to the hydrodynamic effects. To this end, it should be clear that each added inertia matrix $\mathbb{I}_{i j}^{f}$ is symmetric and, hence, has 6 independent components. Further, one also has that $\mathbb{I}_{i j}^{f}=\mathbb{I}_{j i}^{f}$. This symmetry reflects a reciprocity in the effects two submerged ellipses $\mathcal{B}_{i}$ and $\mathcal{B}_{j}$ have on each other due to the hydrodynamic coupling. m) Kinetic Energy of the Solid-Fluid System: By virtue of (3) and (5), the kinetic energy $T$ in (2) can be rewritten as

$$
T=\frac{1}{2} \xi_{i}^{T} \mathbb{I}_{i j} \xi_{j}, \quad i, j=0,1,2,
$$

where $\mathbb{I}_{i i}=\mathbb{I}_{i}^{s}+\mathbb{I}_{i i}^{f}$ and $\mathbb{I}_{i j}=\mathbb{I}_{i j}^{f}$ for $i \neq j$. Note that, although there is an analogy between $\mathbb{I}_{i}^{s}$ and $\mathbb{I}_{i j}^{f}$, they are fundamentally distinct. For example, in translation, unlike the body's actual mass, the added mass depends on the direction of the motion.

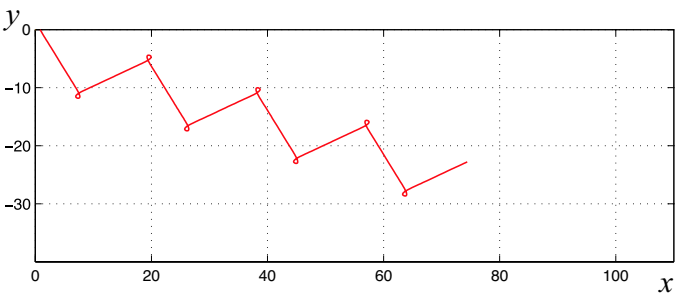

(a)

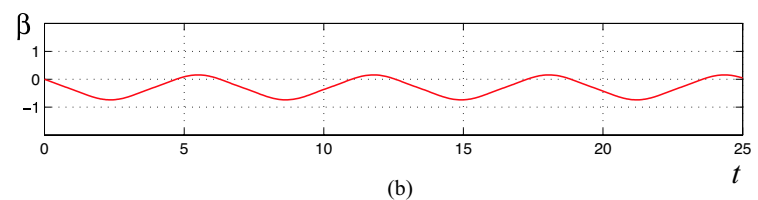

Fig. 4. Forward motion of the neutrally buoyant articulated body due to shape changes $\theta_{1}=-\cos (t)$ and $\theta_{2}=\sin (t)$.
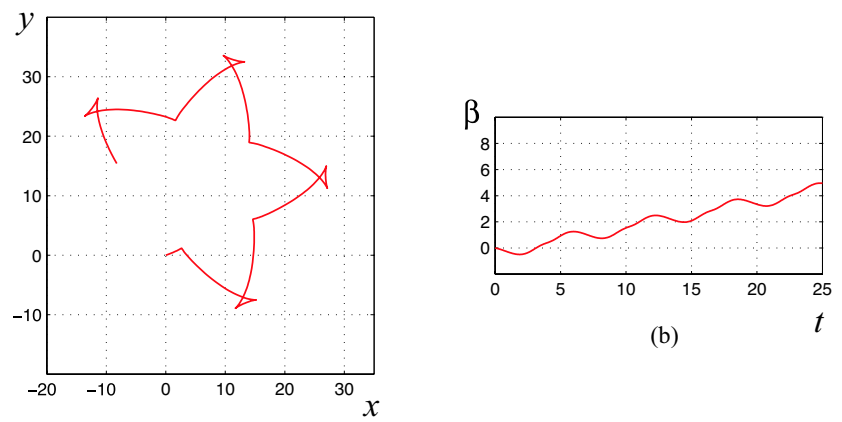

(a)

Fig. 5. Turning motion of the neutrally buoyant articulated body due to shape changes $\theta_{1}=1-\cos (t)$ and $\theta_{2}=-1+\sin (t)$.

\section{The Lagrange-D'Alembert PRInCiPle}

For the neutrally buoyant articulated body, the Lagrangian function $L$ is equal to the kinetic energy $T$ given in (7), and the Lagrange-d'Alembert variational principle requires that

$$
\delta \int_{t_{0}}^{t_{1}} L \mathrm{~d} t+\int_{t_{0}}^{t_{1}}\left(\tau_{1} \cdot \delta \theta_{1}+\tau_{2} \cdot \delta \theta_{2}\right)=0,
$$

for all variations $\left(\delta \theta_{1}, \delta \theta_{2}, \delta \beta, \delta x, \delta y\right)$ that vanish at the end points $t_{0}$ and $t_{1}$. The associated equations of motion can be written concisely as in (10-11). To see this, we first introduce a momentum-like quantity $\mu_{i}$ associated to each $\mathcal{B}_{i}$ and expressed relative to $\mathcal{B}_{i}$-fixed frame

$$
\mu_{i}=\sum_{j=1}^{3} \mathbb{I}_{i j} \xi_{j}
$$


We also define the momentum $\mu_{s}$ of the solid-fluid system relative to the $\mathcal{B}_{0}$-fixed frame as follows $\mu_{s}=\mu_{0}+\widetilde{\mu_{1}}+\widetilde{\mu_{2}}$, where $\widetilde{\mu_{\alpha}}, \alpha=1,2$ correspond to the transformed $\mu_{\alpha}$ from their respective body-fixed frames to the $\mathcal{B}_{0}$-fixed frame. ${ }^{1}$ We then rewrite $\mu_{\alpha}$ as $\mu_{\alpha}=\left(\Pi_{\alpha}, P_{\alpha}\right)^{T}$ where $\Pi_{\alpha}$ and $P_{\alpha}$ denote, respectively, the angular and linear momenta of $\mathcal{B}_{\alpha}$ in the $\mathcal{B}_{\alpha}$-fixed frame. Likewise, we write $\mu_{s}=\left(\Pi_{s}, P_{s}\right)^{T}$. To this end, the equations associated with (8) can be expressed in the form

$$
\begin{aligned}
\dot{\Pi}_{s} & =P_{s} \times v_{0}, \\
\dot{P}_{s} & =P_{s} \times \Omega_{0} .
\end{aligned}
$$

together with

$$
\begin{aligned}
& \dot{\Pi}_{1}=P_{1} \times v_{1}+\tau_{1}, \\
& \dot{\Pi}_{2}=P_{2} \times v_{2}+\tau_{2},
\end{aligned}
$$

That is, we have a determinate system of 5 equations that govern the motion $\left(\theta_{1}(t), \theta_{2}(t), \beta(t), x(t), y(t)\right)$. A derivation of a more general form of these equations is detailed in [6].

n) Conservation of Total Momentum: Let $\widetilde{\mu_{s}}$ be the total momentum written relative to the fixed inertial frame $\left\{\mathbf{e}_{k}\right\}$. One can verify by taking the time derivative of $\widetilde{\mu_{s}}$ and invoking (10) that $\dot{\overrightarrow{\mu_{s}}}=0$, which implies that $\widetilde{\mu_{s}}$ is a conserved quantity or constant of motion.

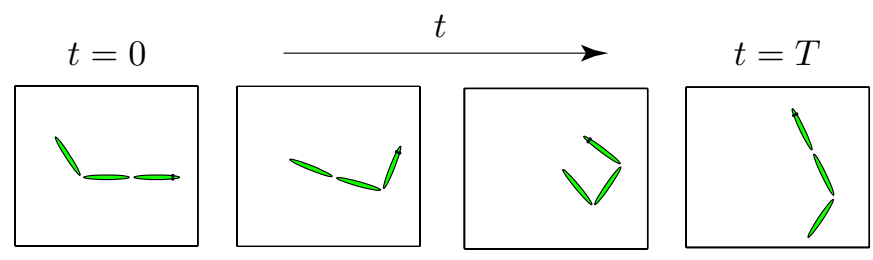

Fig. 6. Turning gait over one period $T=2 \pi$ due to shape changes $\theta_{1}=1-\cos (t)$ and $\theta_{2}=-1+\sin (t)$ (see Figure 5).

\section{Motion Planning}

We investigate the following problem: among all possible shape changes that steer the articulated body from a given initial position and orientation to a desired final position and orientation, find the optimal ones.

o) Solvability and Controllability: The problem is to find the optimal trajectories in $\mathfrak{X}$ that steer $\left(\beta\left(t_{0}\right), x\left(t_{0}\right), y\left(t_{0}\right)\right)$ to $\left(\beta\left(t_{1}\right), x\left(t_{1}\right), y\left(t_{1}\right)\right)$, see Figure 3 . For some given initial and final conditions, there may be no trajectories that achieve the desired motion. In this case the optimization problem has no solution.

In $\S \mathrm{VI}$, we provide numerical evidence that, starting from rest, forward and steering motions $(\beta(t), x(t), y(t))$ can be achieved by prescribing periodic shape changes $\left(\theta_{1}(t), \theta_{2}(t)\right)$ and integrating (10), see Figures 4 and 5 . This suggests that the problem is controllable or, at least, controllable in some finite regions of the configuration space. For a rigorous proof of controllability, one needs to appeal to the Ambrose-Singer theorem ([1]) which gives sufficient

\footnotetext{
${ }^{1}$ One cannot assert that $\mu_{s}$ is the total momentum of the system, which in this problem is indeterminate because the fluid has an infinite domain. Traditionally, $\mu_{s}$ was known as the "impulse" (see [8, Chapter 6]).
}

conditions for every net motion to be realized (this theorem is a restatement of a theorem of [4], now familiar to people in control theory). Such undertaken, although very important, is beyond the scope of the present paper.

p) The Optimization Problem: The shape variables $\left(\theta_{1}, \theta_{2}\right)$ are controlled by the input torques $\left(\tau_{1}, \tau_{2}\right)$. Therefore, we consider $\left(\tau_{1}, \tau_{2}\right)$ to be the control variables and $\left(\theta_{1}, \theta_{2}, \beta, x, y\right)$ to be the state variables which we denote by $q$ for brevity. The optimization problem can then be stated as follows. Given the boundary conditions $q\left(t_{0}\right)=q_{0}$, and $q\left(t_{1}\right)=q_{1}$, find $\left(\tau_{1}, \tau_{2}\right)$ that minimize the cost function

subject to

$$
\int_{t_{0}}^{t_{1}} C\left(q, \dot{q}, \tau_{1}, \tau_{2}\right) \mathrm{dt}
$$

$$
\begin{aligned}
& \delta \int_{t_{0}}^{t_{1}} L(q, \dot{q}) \mathrm{d} t+\int_{t_{0}}^{t_{1}}\left(\tau_{1} \cdot \delta \theta_{1}+\tau_{2} \cdot \delta \theta_{2}\right) \mathrm{d} t \\
&+p_{0} \cdot \delta q_{0}-p_{1} \cdot \delta q_{1}=0
\end{aligned}
$$

for all arbitrary variations $\delta q$. That is, the Lagranged'Alembert principle (8) is restated in (13) without the apriori assumption that the variations vanish at the end points $t_{0}$ and $t_{1}$. Rather, this condition is imposed using the boundary constraints

$$
\delta q_{0}=q\left(t_{0}\right)-q_{0}=0, \quad \delta q_{1}=q\left(t_{1}\right)-q_{1}=0,
$$

and their associated Lagrange multipliers

$$
p_{0}=\left.\frac{\partial L}{\partial \dot{q}}\right|_{t_{0}}, \quad p_{1}=\left.\frac{\partial L}{\partial \dot{q}}\right|_{t_{1}} .
$$

q) The Discrete Optimization Problem: Traditional methods in optimal control such as the multiple shooting (see, e.g., [19]) or the collocation methods (see [20]) rely on a direct integration or fulfillement of (10-11) at certain grid points. The corresponding solutions do not respect, in general, the conservation laws that the equations of motion satisfy, such as the conservation of total momentum in the present problem. To circumvent this difficulty, we use a novel method devised by [5] where the main idea is to discretize the cost function (12) and the variational principle (13) directly using global discretization of the states and the controls. To this end, a path $q(t)$, where $t \in\left[t_{0}=0, t_{1}=1\right]$, is replaced by a discrete path $q_{d}:\{0, h, 2 h, \ldots, N h=1\}$, $N \in \mathbb{N}$. Here, $q_{d}(n h):=q_{n}$ is viewed as an approximation to $q\left(t_{n}=n h\right), n \in \mathbb{N}$ and $n \leq N$. Similarly, the continuous torques $\tau_{\alpha}, \alpha=1,2$ are approximated by discrete torques $\tau_{\alpha d}$ such that $\tau_{\alpha n}=\tau_{\alpha d}(n h)$.

The cost function (12) is approximated on each time interval $[n h,(n+1) h]$ by

$$
C_{d}\left(q_{n}, q_{n+1}, \tau_{\alpha n}, \tau_{\alpha n+1}\right) \approx \int_{n h}^{(n+1) h} C\left(q, \dot{q}, \tau_{\alpha}\right) \mathrm{d} t
$$

which yields the discrete cost function

$$
J_{d}\left(q_{d}, \tau_{\alpha d}\right)=\sum_{n=0}^{N-1} C_{d}\left(q_{n}, q_{n+1}, \tau_{\alpha n}, \tau_{\alpha n+1}\right) .
$$



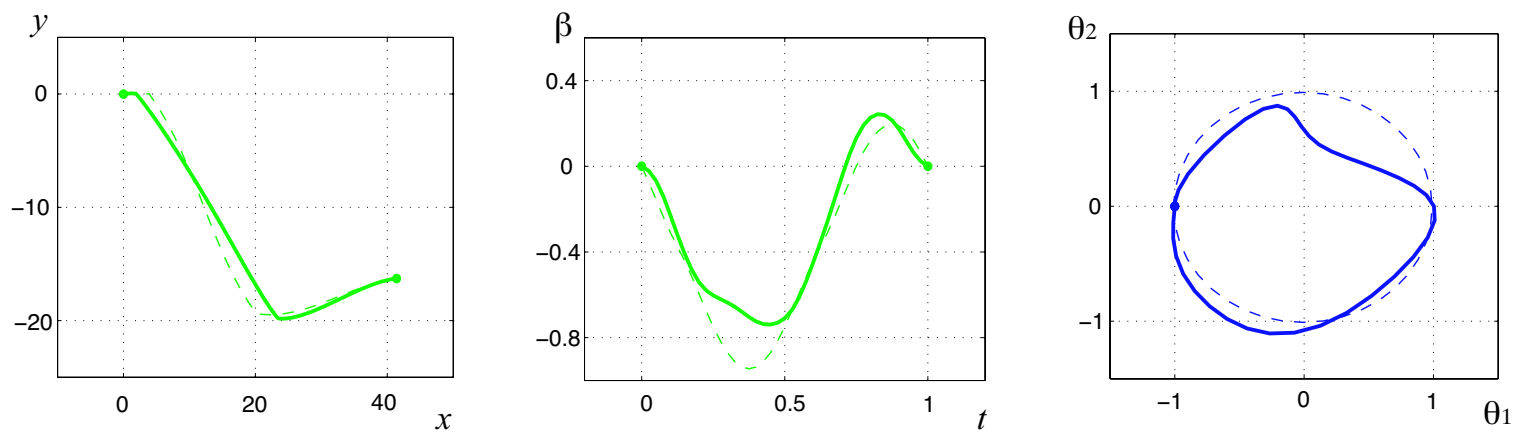

Fig. 7. Optimal solution that produce a net forward motion from an initial position $\left(x_{0}, y_{0}\right)$ to a desired final position $\left(x_{1}, y_{1}\right)$. The boundary conditions are shown as solid dots. The solid lines correspond to the optimal solution while the dashed lines represent the initial guess.

The action integral (13) is approximated on each time interval $[n h,(n+1) h]$ by a discrete Lagrangian

$$
L_{d}\left(q_{n}, q_{n+1}\right) \approx \int_{n h}^{(n+1) h} L(q, \dot{q}) \mathrm{d} t .
$$

We also approximate

$\int_{t_{n}}^{t_{n+1}} \tau_{\alpha} \cdot \delta \theta_{\alpha} \approx \tau_{\alpha n}^{-} \cdot \delta \theta_{\alpha n}+\tau_{\alpha n}^{+} \cdot \delta \theta_{\alpha n+1}, \quad \alpha=1,2$,

where $\tau_{\alpha n}^{-}$and $\tau_{\alpha n}^{+}$are called left and right discrete torques, respectively. The discrete version of (13) requires one to find paths $\left\{q_{n}\right\}_{n=0}^{N}$ such that for all variations $\left\{\delta q_{n}\right\}_{n=0}^{N}$, one has

$$
\begin{aligned}
\delta \sum_{n=0}^{N-1} L_{d}\left(q_{n}, q_{n+1}\right) & +\sum_{n=0}^{N-1} \sum_{\alpha=1}^{2} \tau_{\alpha n}^{-} \cdot \delta \theta_{\alpha n}+\tau_{\alpha n}^{+} \cdot \delta \theta_{\alpha n+1} \\
& +p_{0} \cdot \delta q_{0}+p_{1} \cdot \delta q_{1}=0 .
\end{aligned}
$$

The discrete variational principle (16) yields the following equality constraints

$$
\begin{aligned}
D_{2} L_{d}\left(q_{n-1}, q_{n}\right)+D_{1} L_{d}\left(q_{n}, q_{n+1}\right)+\tau_{n-1}^{+}+\tau_{n}^{-} & =0 \\
p_{0}+D_{1} L_{d}\left(q_{0}, q_{1}\right)+\tau_{0}^{-} & =0 \\
-p_{1}+D_{2} L_{d}\left(q_{N-1}, q_{N}\right)+\tau_{N-1}^{+} & =0
\end{aligned}
$$

where $D_{1}$ and $D_{2}$ denote the derivatives with the respect to the first and second argument, respectively, and $q_{n}=\left(\theta_{1 n}, \theta_{2 n}, \beta_{n}, x_{n}, y_{n}\right), \tau_{n}=\left(\tau_{1 n}, \tau_{2 n}, 0,0,0\right), n=$ $1, \ldots, N$.

r) Summary and Remarks: The discrete nonlinear optimization problem can be stated as follows: find the discrete torques $\left\{\tau_{\alpha n}\right\}_{n=0}^{N}$ and paths $\left\{q_{n}\right\}_{n=0}^{N}$ that minimize the discrete cost function (15) subject to the nonlinear constraints (17) and the boundary conditions (14). We note the following:

1) Additional constraints such as actuators limits can be easily handled by this method: one would add to the list of constraint equations (14) and (17) the inequality constraints corresponding to the limits on $\tau_{n}$.

2) The method presented here is suitable for optimization over a fixed time interval $\left[t_{0}, t_{1}\right]$. Work on time optimal control is under development and would be interesting for the fish problem.

\section{NUMERICAL RESUlTS}

For simplicity, assume that the three ellipses are identical (see Figure 2) and let $a=10, b=1 c=2$ and $\rho_{f}=1 / \pi$. Further, assume that the added inertias associated with a given ellipse are not affected by the presence of the other ellipses. ${ }^{2}$ This assumption is capable of capturing qualitatively the correct dynamics (as demonstrated in [6]). To this end, the inertia matrices are given by $\mathbb{I}_{11}=\mathbb{I}_{22}=\mathbb{I}_{33}=\mathbb{I}$ and $\mathbb{I}_{i j}=0$, for $i \neq j$. In addition, $\mathbb{I}$ is a diagonal matrix with non-zero diagonal entries

$$
j=I+I^{f}, \quad m_{1}=m+m_{1}^{f}, \quad m_{2}=m+m_{2}^{f},
$$

where the body moment of inertia $I$ and mass $m$ are given in $\S$ III, while the added inertias $I^{f}, m_{1}^{f}$ and $m_{2}^{f}$ due to the fluid effects are given by (see, for example, [15, Chapter 4])

$I^{f}=\frac{1}{8} \rho_{\mathcal{F}} \pi\left(a^{2}-b^{2}\right)^{2}, m_{1}^{f}=\rho_{\mathcal{F}} \pi b^{2}, m_{2}^{f}=\rho_{\mathcal{F}} \pi a^{2}$.

s) Direct Numerical Integration: Starting from rest, we prescribe periodic shape changes $\left(\theta_{1}(t), \theta_{2}(t)\right)$ and compute the resulting $(\beta(t), x(t), y(t))$ by integrating (10) using a standard $4^{\text {th }}$ order Runge-Kutta integration scheme with constant time steps.

Figure 4 shows a net forward motion of the three-link fish in the $\left(\mathbf{e}_{1}, \mathbf{e}_{2}\right)$-plane due to shape changes $\theta_{1}=-\cos (t)$ and $\theta_{2}=\sin (t)$. In Figure 5, the three-link fish is shown to turn counterclockwise in the $\left(\mathbf{e}_{1}, \mathbf{e}_{2}\right)$ plane due to shape changes $\theta_{1}=1-\cos (t)$ and $\theta_{2}=-1+\sin (t)$. Snapshots of the turning maneuvers over one period $T=2 \pi$ of shape changes are shown in Figure 6.

t) Implementation of the Discrete Optimization Scheme: To approximate the relevant integrals in (12) and (13), we use the midpoint rule; that is, in (15), we set

$$
\begin{aligned}
& C_{d}\left(q_{n}, q_{n+1}, \tau_{\alpha n}, \tau_{\alpha n+1}\right)= \\
& \quad h C\left(\frac{q_{n}+q_{n+1}}{2}, \frac{q_{n+1}-q_{n}}{h}, \frac{\tau_{\alpha n}+\tau_{\alpha n+1}}{2}\right), \alpha=1,2,
\end{aligned}
$$

and, in (16), we use

$$
L_{d}\left(q_{n}, q_{n+1}\right)=h L\left(\frac{q_{n}+q_{n+1}}{2}, \frac{q_{n+1}-q_{n}}{h}\right),
$$

\footnotetext{
${ }^{2}$ Note that this assumption is accurate when the ellipses $\mathcal{B}_{i}$ are placed a large distance apart.
} 

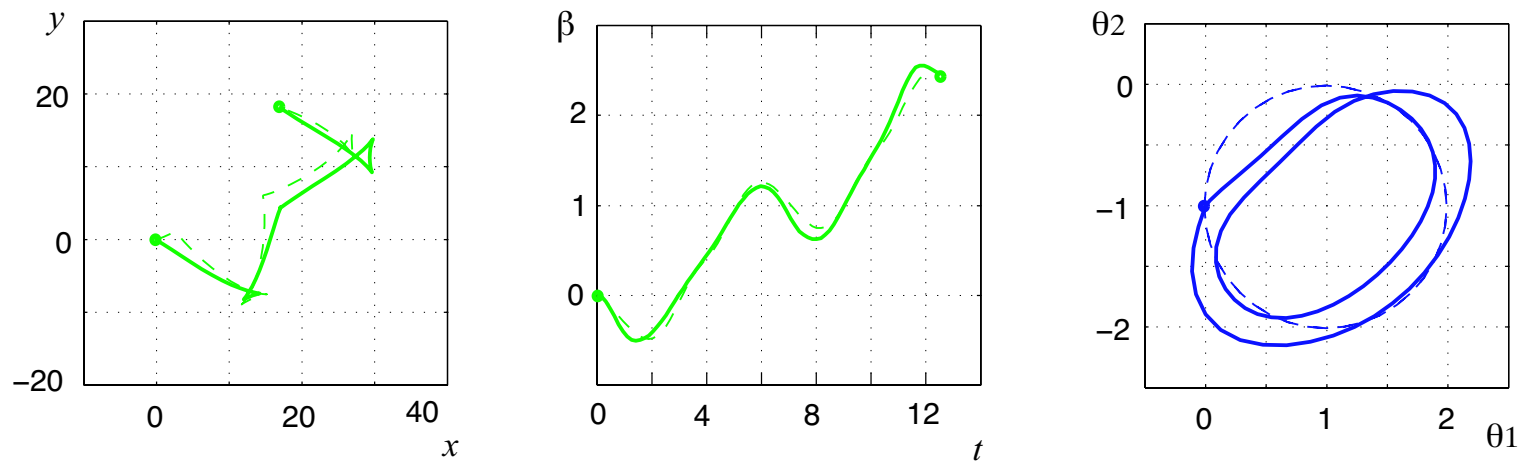

Fig. 8. Optimal solution that steers and propels the system from $\left(\beta_{0}, x_{0}, y_{0}\right)$ to $\left(\beta_{1}, x_{1}, y_{1}\right)$. The boundary conditions are shown as solid dots. The solid lines correspond to the optimal solution while the dashed lines represent the initial guess.

while

$$
\tau_{\alpha n}^{-}=\tau_{\alpha n}^{+}=\frac{h}{4}\left(\tau_{\alpha n}+\tau_{\alpha n+1}\right) \quad \alpha=1,2 .
$$

We solve the discrete optimization problem of finding $\left\{\tau_{\alpha n}\right\}_{n=0}^{N}$ and $\left\{q_{n}\right\}_{n=0}^{N}$ that minimize (15) subject to the nonlinear constraints (17) and the boundary conditions (14) using a built-in Matlab function for sequential quadratic programming. This optimization method is only local: based on the choice of an initial guess, the optimal solution is determined by means of infinitesimal variations.

u) Optimization Results: In the examples presented in this section, the goal is to minimize the control effort, hence the cost function (12) is taken to be

$$
J\left(q, \tau_{1}, \tau_{2}\right)=\int_{t_{0}}^{t_{1}}\left(\tau_{1}^{2}+\tau_{2}^{2}\right) \mathrm{dt} .
$$

Figure 7 shows the locally optimal solution that produces a net forward motion from $\left(x_{0}, y_{0}\right)$ to $\left(x_{1}, y_{1}\right)$ in the $\left(\mathbf{e}_{1}, \mathbf{e}_{2}\right)$ plane. Interestingly, the optimal shape changes trace a trajectory of non-regular shape because of the nonlinear nature of the problem. It is worth noting that in order to obtain the initial guesses in Figure 7, we take the trajectories of Figure 4 over the time intervals $[0, T], T=2 \pi$, and impose small perturbations on these portions. Likewise, the initial guess in Figure 8 corresponds to an arbitrary perturbation on the trajectories of Figure 5 over $[0,2 T]$. One does not obtain a repeated pattern of shape changes when optimizing over several time periods (as shown in Figure 8 over two periods). This observation leads to the interesting question whether to optimize over one period of shape changes or over the whole time interval of the desired locomotion.

\section{CLOSING REMARKS}

This paper considers an articulated body that can undergo shape changes and is submerged in a perfect fluid. We show that the submerged body is able to propel and steer itself by changing its shape, and we investigate the optimal shape changes that produce a desired net locomotion. This problem is formulated as a constrained optimization problem where the nonlinear constraints correspond to the equations governing the motion of the solid-fluid system and are defined via the Lagrange-d'Alembert variational principle.

\section{REFERENCES}

[1] Ambrose, W. and I.M. Singer [1953], A theorem on holonomy, Trans. AMS, 75, 428-453.

[2] Cortés, J., S. Martínez, J. P. Ostrowski and K. A. McIsaac [2001], Optimal gaits for dynamic robotic locomotion Intern. J. of Robotics Research, 20, 707-728.

[3] Childress, S. [1981], Mechanics of swimming and flying, Cambridge University Press, NY.

[4] Chow, W.L. [1939], Uber systeme van linearen partiellen differentialgleichungen erster ordnung, Math. Ann, 117, 98-105.

[5] Junge, O., Marsden, J.E. and Ober-Blöbaum, S. [2005], Discrete optimal control, Proc. IFAC Conf., Prague.

[6] Kanso, E., J.E. Marsden, C.W. Rowley and J. Melli-Huber [2005], Locomotion of articulated bodies in a perfect fluid, J. Nonlinear Science, 15:255-289.

[7] Kelly, S. D. [1998], The Mechanics and Control of Robotic Locomotion with Applications to Aquatic Vehicles, $\mathrm{PhD}$ thesis, California Institute of Technology.

[8] Lamb, H. [1932], Hydrodynamics. Dover, NY, sixth edition.

[9] Liao, J.C., D.N. Beal, G.V. Lauder and M.S. Triantafyllou [2003], Fish exploiting vortices decrease muscle activity Science, 302, 1566-1569.

[10] Lighthill, J. [1975], Mathematical Biofluiddynamics. Society for Industrial and Applied Mathematics, PA.

[11] Melli-Huber, J.B., D. Rufat, C.W. Rowley [2005], Motion Planning for an Articulated Body in a Perfect Fluid, (in preparation).

[12] Montgomery, R. [1990], Isoholonomic Problems and Some Applications, Commun. Math. Phys. 128: 565-592.

[13] Morgansen K. A., V. Duindam, R. J. Mason, J. W. Burdick and R. M. Murray [2001] Nonlinear control methods for planar carangiform robot fish locomotion, In Proc. IEEE International Conference on Robotics and Automation, pp. 427-434.

[14] Müller, U. K. [2003], Fish'n Flag, Science 302, pp. 1511-1512.

[15] Newman, J. N [1977], Marine Hydrodynamics. MIT press, Cambridge, Ma.

[16] Ostrowski J. P., J. P. Desai and V. Kumar [2000], Optimal gait selection for nonholonomic locomotion systems Intern. J. of Robotics Research, 19, 225-237.

[17] Radford, J. and J. Burdick [1998], Local motion planning for nonholonomic control systems evolving on principal bundles, In Proc. Mathematical Theory of Networks and Systems.

[18] Radford, J. [2003], Symmetry, Reduction and Swimming in a Perfect Fluid, PhD thesis, California Institute of Technology.

[19] Stoer, J. and R. Bulirsch [2002], Introduction to numerical analysis, Springer, New York, 12 xvi+744.

[20] von Stryk, O. [1993], Numerical solution of optimal control problems by direct collocation, Optimal control (Freiburg, 1991), Int. Ser. Numer. Math., Birkhäuser, Basel, 129-143.

[21] Taylor, G.I. [1952], Analysis of the swimming of long and narrow animals, Proc. Roy. Soc. London A, 214(1117): 158-183.

[22] Webb, P.W. [1991], Composition and mechanics of routine swimming of rainbow trout, Oncorhynchus mykiss. Can. J. Fish. Aquat. Sci. 48, 583-590.

[23] Wu, T.Y. [1971], Hydrodynamics of swimming fishes and cetaceans, Adv. appl. math. 11, 1-63. 